\title{
AOR
}

Selected Papers of \#AolR2021:

The 22nd Annual Conference of the

Association of Internet Researchers

Virtual Event / 13-16 Oct 2021

\section{INTO THE BELLY OF THE BEAST: THE RESEARCH ON SOCIAL MEDIA AND COVID-19 MISINFORMATION IN 2020}

\author{
Nicola Righetti \\ University of Vienna \\ Luca Rossi \\ IT University of Copenhagen \\ Giada Marino \\ University of Sassary
}

\section{Introduction}

In this paper we present some preliminary findings from an ongoing research (Righetti, Rossi, Marino, 2021) on a comprehensive corpus of 378 interdisciplinary studies about misinformation and COVID-19 published in 2020, focusing on the role of social media platforms in spreading and countering mis- and disinformation.

From the health of democracy to the health of people, after the major wave of research on "fake news" following the 2016 U.S. Presidential elections (Righetti, 2021), the COVID-19 pandemic has probably been the event that attracted most of the research on the topic.

Changing the everyday lives of people around the world, the threatening and unknown virus has led to an explosive stream of information, including unsubstantiated, misleading, and false information (Brennen et al., 2020) which led the WHO to use the term "infodemic" to label this excessively prolific, confused and potentially harmful flow of information.

Social media platforms have been into the belly of the infodemic beast. Pointed out as one of the main means for spreading groundless and harmful disinformation, they have also implemented a series of countermeasures to curb misinformation and connect

Suggested Citation (APA): Righetti, N., Rossi, L., Marino, G. (2021, October). Into the belly of the beast: The research on social media and covid-19 misinformation in 2020. Paper presented at AolR 2021: The 22nd Annual Conference of the Association of Internet Researchers. Virtual Event: AolR. Retrieved from http://spir.aoir.org. 
people to official information sources, becoming an important tool for government to debunk fake news and promote health information (Lovari, 2020).

Here we aim at answering the following research questions:

RQ1) Which social media platforms have been taken into account by the research on COVID-19 misinformation in 2020?

RQ2) What methods have been used by these studies?

RQ3) Which perspectives on misinformation and social media emerge from them?

\section{Data and Method}

Following the PRISMA guidelines for systematic reviews (Moher et al., 2009) we collected, screened and analyzed a comprehensive interdisciplinary corpus of 565 papers on COVID-19 misinformation published in 2020 (Fig. 1). The collected data set can be defined largely complete, considering the records for the same keywords:obi]: registered in the official COVID-19 literature research service of WHO $(\mathrm{N}=484)^{1}$.

Fig. 1. PRISMA flow chart
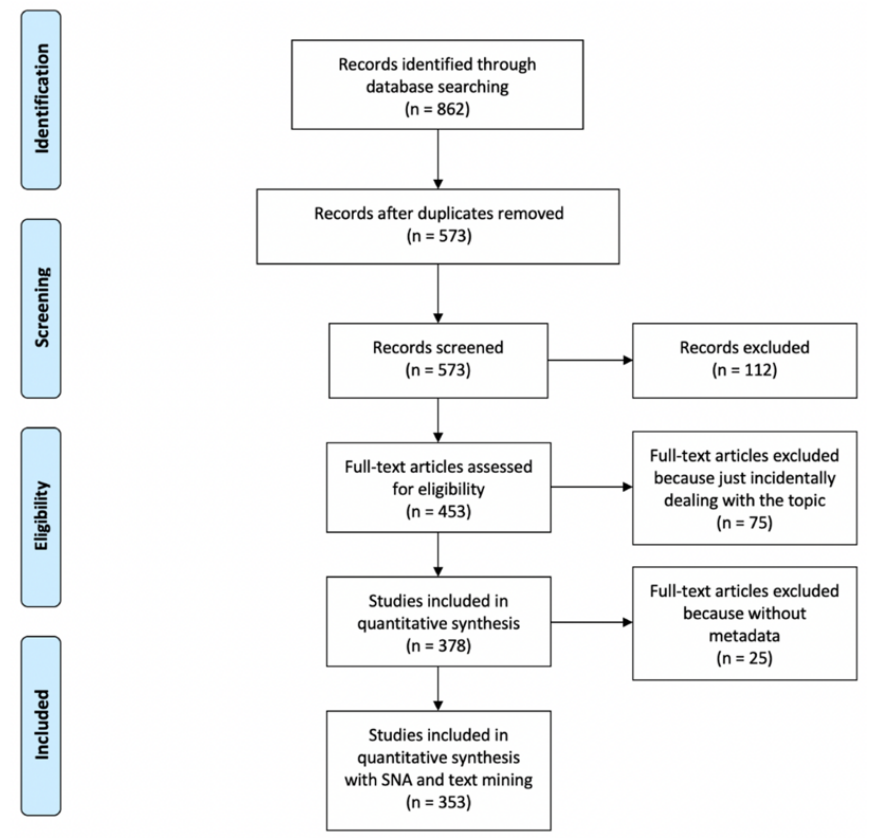

\footnotetext{
${ }^{1}$ COVID OR COVID-19 OR coronavirus AND misinformation OR disinformation OR malinformation OR "problematic information" OR "fake news" OR "information disorder" OR infodemic OR conspiracy OR rumor OR hoax.
} 
Of 453 articles assessed for eligibility, 75 studies were excluded considering that they just incidentally mention the topic $(\mathrm{N}=378)$. The papers were read by three coders and the main information annotated according to a coding scheme on methodological and substantive questions ${ }^{2}$. This data set was used to answer the above-mentioned research questions by using a quantitative and qualitative approach.

\section{Findings}

The most popular and accessible social media platforms - Twitter and

Facebook - are the most mentioned in the investigated papers, followed by

WhatsApp, Instagram and YouTube. Less mainstream platforms (e.g.:

Telegram and Reddit) are less represented, and non-Western platforms (e.g.:

WeChat and Odnoklassniki) are markedly underrepresented (Fig. 2).

Figure 2. Platforms mentioned in the studies.

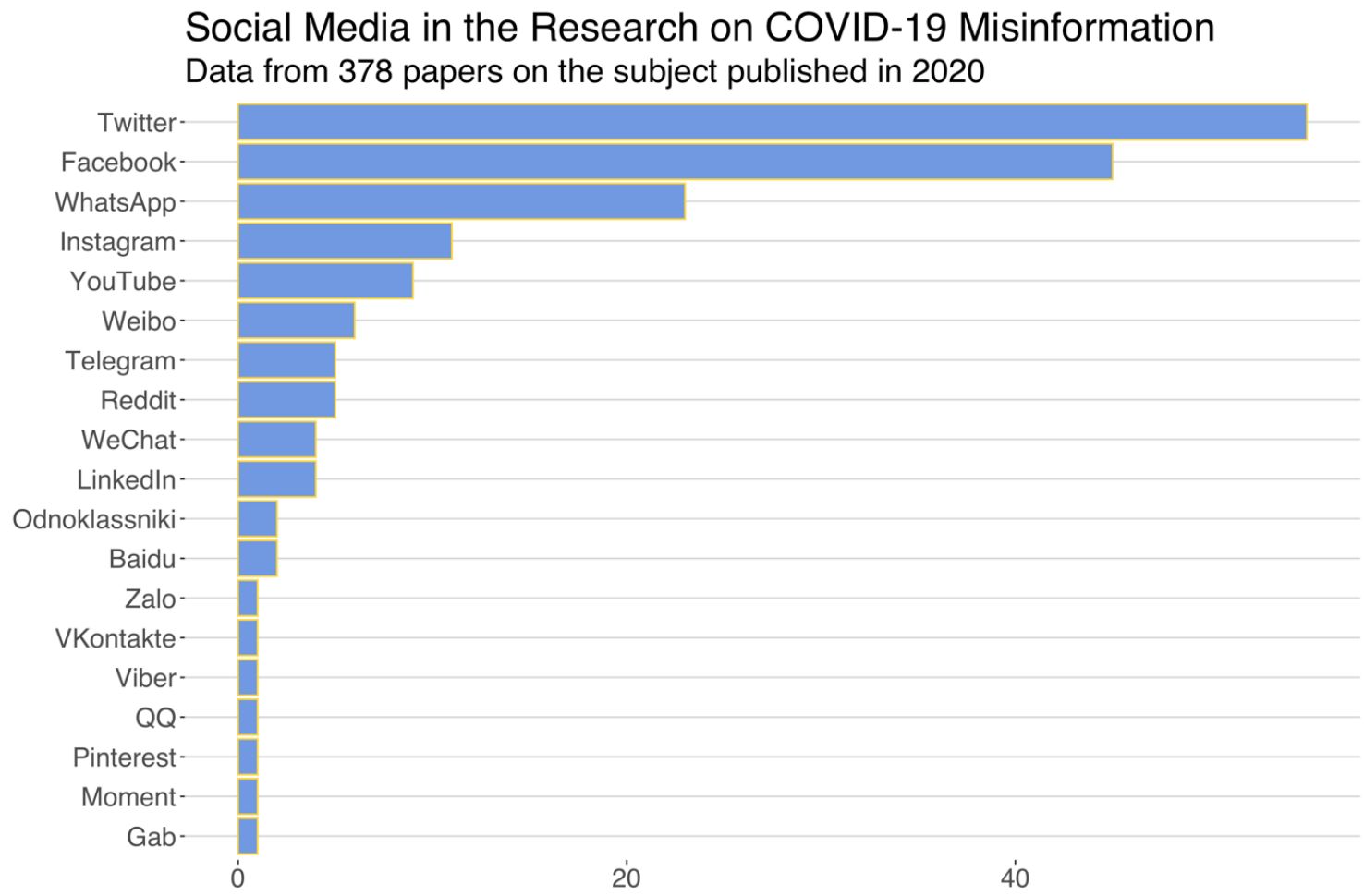

As shown in the next plot (Fig. 3), most of the studies used social media to distribute a survey, therefore collecting a convenience sample of social media users, or make reference to social media in a survey. It can also be seen the impact of data availability on the methods used by researchers: for instance,

\footnotetext{
${ }^{2}$ Among the categories taken into consideration: methods, units of analysis, sample size, country, social media platforms, and main findings.
} 
social network analysis or natural language processing (NLP) require data generally not available for instant messaging platforms such as WhatsApp, so they are not used to study this platform.

Many perspectives and findings emerged in the research. Here we can just make a few examples, highlighting three crucial topics - prevalence of misinformation, countermeasures, and the role of social media communication to bring out the polyphony of scholar voices. About misinformation prevalence, for instance, some study found just minor misinformation (Prabhakar \& Prasad, 2020), while others a relevant and impactful flow of misleading content ( $\mathrm{Li}$ et al. 2020).

Figure 3. Methods used in the studies.

Methods by Social Media in the Research on COVID-19 Misinformation Data from 378 papers on the subject published in 2020

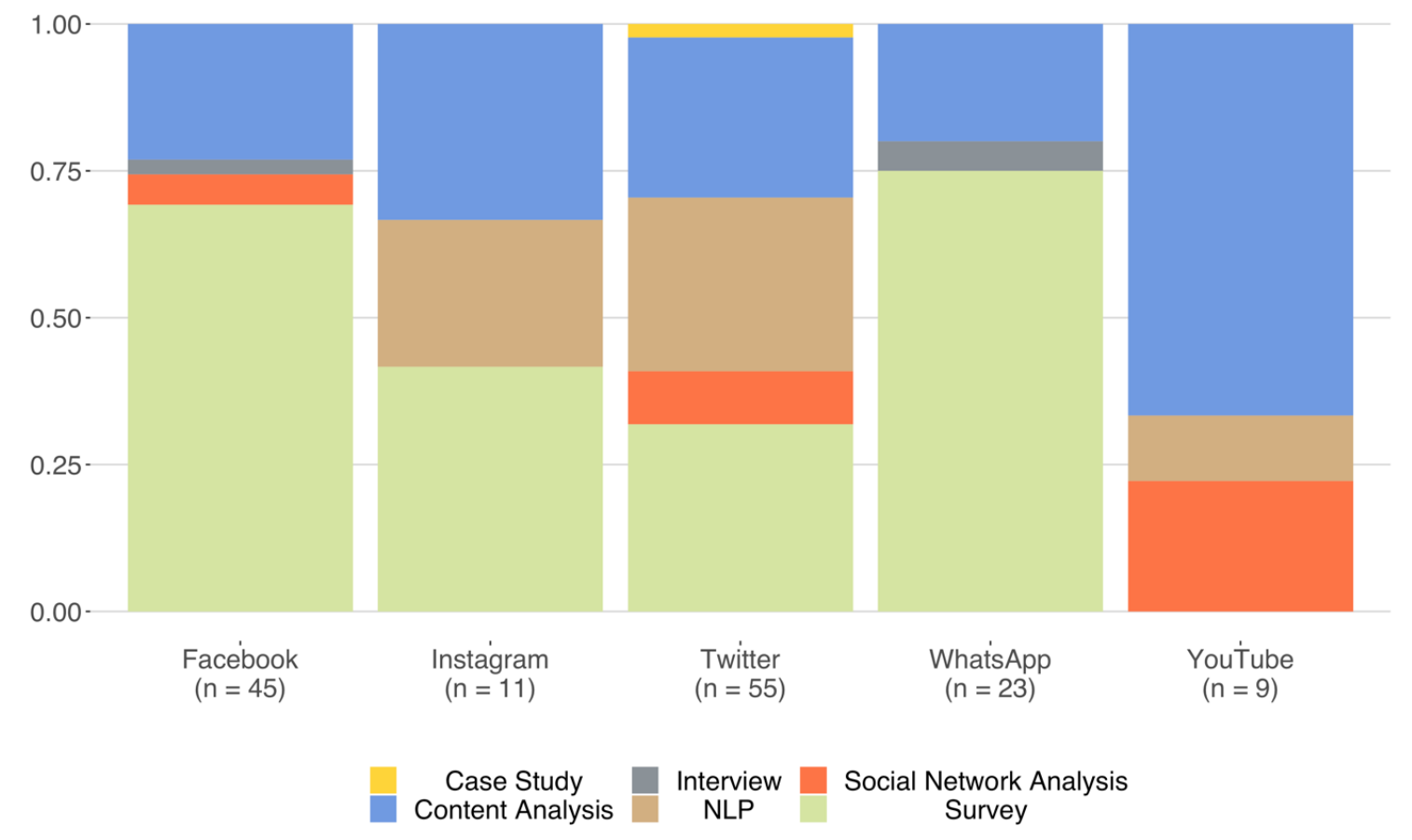

With reference to moderating practices, some scholars have observed they could even strengthen conspiracy beliefs, and that banned content keeps circulating on alternative platforms (Bruns, Harrington, \& Hurcombe, 2020), while others have even gone so far as to suggest, as a viable solution to counter disinformation, collaboration with platforms to imprison who spread fake news (Alvarez-Risco, 2020). Finally, although many papers focus on the role of social media in the infodemic, others have observed that they can also be used against it, for instance thanks to celebrities sharing quality information with their followers (Mututwa, 2020), or by official agencies to disseminate pandemic-related information (Liu \& Tong, 2020). 


\section{Conclusions}

The preliminary results we have sketched, show that research on COVID-19 misinformation reproduces a well-known trend of differentiated attention to social media platforms based on both popularity among users and ease of access to data by scholars. This points out the importance for researchers to get wider access to social media data due to the constrains that, otherwise, will inevitably limit research based on just a few platforms.

About methods, online survey distributed via social media has been a very popular approach, due to the urgency to understand what was going on with the pandemic in the information ecosystem, along with the relevance of digital platforms for the spreading of misinformation, and the ease to collect data through them. However, this approach has several limitations, mainly because it relies on convenience samples which can lead to a partial view on the issue.

Beside limitations, the research has made evident a wide range of perspective on problematic information, and sometimes diverging point of views. It is therefore important to summarize the studies already made on the topic to favor the development of the research, also pointing out limitations to overcome, viable solutions, and new hypotheses to test.

\section{References}

Alvarez-Risco, A., Mejia, C. R., Delgado-Zegarra, J., Del-Aguila-Arcentales, S., ArceEsquivel, A. A., Valladares-Garrido, M. J., ... \& Yáñez, J. A. (2020). The Peru approach against the COVID-19 infodemic: insights and strategies. The American journal of tropical medicine and hygiene, 103(2), 583-586.

Bruns, A., Harrington, S., \& Hurcombe, E. (2020). 'Corona? 5G? or both?': the dynamics of COVID-19/5G conspiracy theories on Facebook. Media International Australia, 177(1), 12-29.

Li, H. O. Y., Bailey, A., Huynh, D., \& Chan, J. (2020). YouTube as a source of information on COVID-19: a pandemic of misinformation? BMJ global health, 5(5).

Liu, J. C., \& Tong, E. M. (2020). The relation between official WhatsApp-distributed COVID-19 news exposure and psychological symptoms: Cross-sectional survey study. Journal of medical Internet research, 22(9).

Lovari, A. (2020). Spreading (dis) trust: Covid-19 misinformation and government intervention in Italy. Media and Communication, 8(2), 458-461. 
Moher, D., Shamseer, L., Clarke, M., Ghersi, D., Liberati, A., Petticrew, M., ... \& Stewart, L. A. (2015). Preferred reporting items for systematic review and meta-analysis protocols (PRISMA-P) 2015 statement. Systematic reviews, 4(1), 1-9.

Mututwa, W. (2020). COVID-19 infections on international celebrities: self-presentation and tweeting down pandemic awareness. Journal of Science Communication, 19(5)

Prabhakar Kaila, D., \& Prasad, D. A. (2020). Informational flow on Twitter-Corona virus outbreak-topic modelling approach. International Journal of Advanced Research in Engineering and Technology, 11(3).

Righetti, N., Rossi, L., Marino, G. (2021). The 2020 Research on Problematic Information on the COVID19 Pandemic. A Systematic Literature Review https://osf.io/preprints/socarxiv/fn3cj/

Righetti, N. (2021). Four Years of Fake News. A Quantitative Analysis of the Scientific Literature. First Monday. 